\title{
SOME PROPERTIES OF URYSOHN-STIELTJES INTEGRAL OPERATORS
}

\author{
JÓZEF BANAŚ \\ Department of Mathematics \\ Technical University of Rzeszów \\ W Pola 2, 35-959 Rzeszów, POLAND
}

(Received January 23, 1996 and in revised form December 12, 1996)

\begin{abstract}
We investigate some properties of Urysohn-Stieltjes integral operators. The assumptions will be formulated under which the operators in question transform the space of continuous functions into itself or in the space of functions of bounded variation, are continuous or are compact. The solvability of an integral equation of Urysohn-Stieltjes type will be also discussed
\end{abstract}

KEY WORDS AND PHRASES: Nonlinear integral operator, function of bounded variation. Streltjes integral, and Urysohn-Stieltjes integral equation.

1991 AMS SUBJECT CLASSIFICATION CODE: 47H30.

\section{INTRODUCTION}

It is well-known that integral operators of various types create an important subject of numerous mathematical investigations and are often applicable in mathematical physics, engineering, economics and so on (cf. the following monographs. Burton [1]; Krasnosel'skii et al. [2]; Ladde et al. [3], Tricomi [4]; Zabrejko et al. [5]).

The goal of this paper is to study the integral operators of Urysohn-Stieltjes type with kernel depending on two variables. Obviously operators of this kind contain as the special cases several integral operators frequently considered both in the theory of integral equations and in nonlinear functional analysis. Let us mention, for example, the classical linear integral operators of Fredholm type or the nonlinear integral operators of Hammerstein or Urysohn type considered by Krasnosel'skii et al. [2]; Tricomi [4] and Zabrejko et al. [5], among others.

On the other hand in the literature one can meet several papers or books devoted to the study of integral operators of Stieltjes type with kernels of integrals involved depending mostly on one varıable (see, for example, Bitzer [6]; Chen et al. [7]; Macnerney [8] and Mingarelli [9]).

It is worthwhile to mention that some special cases of the integral operators of Urysohn-Stieltjes type with kernels depending on two variables can be encountered in the theory of differential equations with retarded argument, especially in Myškis sense (cf. Bielecki [10], Dłotko [11, 12], Ladde et al. [3], Lovelady [13] and Myškis [14]).

In this paper we are going to consider several classical properties of the Urysohn-Streltjes integral operators. Particularly, we will study the conditions guaranteeing that operators of this type transform the space of continuous functions into itself, into the space of bounded functions or into the space of functions with bounded variation. We investigate also some other properties of operators in question such as continuity or compactness. The results obtained will be applied to the study of the solvability of an integral equation of Urysohn-Stieltjes type.

The investigations of this paper form a continuation of the study from the paper of Banas and Dronka [15], where we have considered the integral operators of Fredholm-Stieltjes and Hammerstein-Stieltjes type Moreover, we correct some errors made in the above quoted paper 
The results of the present paper generalize several ones obtained previously in the papers of Banaś and Dronka [15], Bielecki [10]; Chen et al. [7]; Dłotko [11, 12]; Krasnosel'skii et al. [2], Ladde et al. [3], Mingarelli [9]; Myškis [14] and Zabrejko et al. [5], among others.

\section{NOTATION DEFINITIONS AND AUXILIARY FACTS}

In this section we present a few auxiliary facts needed in the sequel of the paper.

Let $x$ be a given real function defined on the interval $[a, b]$. Denote by $\stackrel{b}{V}_{a}^{b}$ the variation of $x$ on the interval $[a, b]$. If $\underset{a}{b} x$ is finite we say that $x$ is of bounded variation on the interval $[a, b]$.

The fundamental properties of functions of bounded variation may be found in Dunford and Schwartz [16] and Natanson [17], for example

In the case we consider a function of two variables $u(t, s)=u:[a, b] \times[c, d] \rightarrow \mathbb{R}$ then the symbol $\bigvee_{t=p}^{q} u(t, s)$ indicates the variation of the function $t \rightarrow u(t, s)$ on the interval $[p, q] \subset[a, b]$, where $s$ is an arbitrarily fixed number in $[c, d]$.

In the same way we define the quantity $\mathrm{V}_{s=p}^{q} u(t, s)$.

In what follows let $x, \varphi:[a, b] \rightarrow \mathbf{R}$ be bounded functions. Then, under some additional conditions formulated, for example, in Dunford and Schwartz [16] and Natanson [17], we can define the Stieltjes integral

$$
\int_{a}^{b} x(t) d \varphi(t)
$$

of the function $x$ with respect to the function $\varphi$. In this case we say that $x$ is Stieltjes integrable on the interval $[a, b]$ with respect to $\varphi$.

Let us recollect two basic cases of Stieltjes integrability. Namely, if we assume that $x$ is continuous and $\varphi$ of bounded variation on the interval $[a, b]$ then $x$ is Stieltjes integrable with respect to $\varphi$ on $[a, b]$. Moreover, under the assumption that $x$ and $\varphi$ are of bounded variation on $[a, b]$ the Stieltjes integral $\int_{a}^{b} x(t) d \varphi(t)$ exists if and only if the functions $x$ and $\varphi$ have no common points of discontinuity.

Now we provide a few properties of the Stieltjes integral which will be used further on. The first property is formulated in the following lemma which may be found in Myškis [14] and Natanson [17].

LEMMA 2.1. If $x$ is Stieltjes integrable on the interval $[a, b]$ with respect to a function $\varphi$ of bounded variation then

$$
\left|\int_{a}^{b} x(t) d \varphi(t)\right| \leq\left(\sup _{a \leq t \leq b}|x(t)|\right) \underset{a}{\operatorname{V}} \varphi .
$$

Moreover, the following inequality holds

$$
\left|\int_{a}^{b} x(t) d \varphi(t)\right| \leq \int_{a}^{b}|x(t)| d\left(\mathrm{~V}_{a}^{t} \varphi\right) .
$$

The inequalities between Stieltjes integrals are described in the next two lemmata. The first one comes from Myškis [14].

LEMMA 2.2. Assume that $x_{1}$ and $x_{2}$ are integrable on the interval $[a, b]$ with respect to a nondecreasing function $\varphi$ and $x_{1}(t) \leq x_{2}(t)$ for $t \in[a, b]$. Then

$$
\int_{a}^{b} x_{1}(t) d \varphi(t) \leq \int_{a}^{b} x_{2}(t) d \varphi(t) .
$$


LEMMA 2.3. Let $x$ be a nonnegative function on the interval $[a, b]$ which is Steiltjes integrable on this interval with respect to functions $\varphi_{1}$ and $\varphi_{2}$. If $\varphi_{2}-\varphi_{1}$ is nondecreasing on $[a, b]$ then

$$
\int_{a}^{b} x(t) d \varphi_{1}(t) \leq \int_{a}^{b} x(t) d \varphi_{2}(t) .
$$

The proof is a simple consequence of the definition of the Stieltjes integral and is therefore omitted.

REMARK. In the paper of Banaś and Dronka [15] instead of Lemma 2.3 the following result has been used (cf. Lemma 5 in the mentioned paper):

Let $\mathrm{x}$ be a continuous and nonnegative function on the interval $[a, b]$ and let $\varphi_{1}, \varphi_{2}$ be nondecreasing on $[a, b]$ and such that $\varphi_{1}(a)=\varphi_{2}(a)$ and $\varphi_{1}(t) \leq \varphi_{2}(t)$ for each $t \in[a, b]$. Then

$$
\int_{a}^{b} x(t) d \varphi_{1}(t) \leq \int_{a}^{b} x(t) d \varphi_{2}(t) .
$$

Unfortunately this result is not valid. In order to show this let us take the following functions defined on the interval $[0,1]$ :

$$
\begin{aligned}
& \varphi_{1}(t)=t, \\
& \varphi_{2}(t)=\left\{\begin{array}{lll}
0 \text { for } t=0 \\
1 \text { for } t \in(0,1]
\end{array}\right. \\
& x(t)=\left\{\begin{array}{lll}
0 & \text { for } t \in[0,1 / 2) \\
2 t-1 & \text { for } & t \in[1 / 2,1]
\end{array}\right.
\end{aligned}
$$

Then

$$
\int_{0}^{1} x(t) d \varphi_{1}(t)>0, \int_{0}^{1} x(t) d \varphi_{2}(t)=0 .
$$

By this reason some results given in Banaś and Dronka [15] are not correct. In this paper we provide improved versions of those results.

For our further purposes we will need some properties of the so-called superposition operator (cf. Appell and Zabrejko [18]). In order to define this operator assume that $f:[a, b] \times \mathbb{R} \rightarrow \mathbb{R}$ is a given function. Then to every function $x$ defined on $[a, b]$ we may assign the function $(F x)(t)=$ $f(t, x(t)), t \in[a, b]$. The operator $F$ defined in this way is said to be the superposition operator generated by the function $f(t, x)$.

Now, let us denote by $C[a, b]$ the space of all continuous functions acting from $[a, b]$ into $\mathbb{R}$ with the standard maximum norm.

Then we have the following result which may be found in Appell and Zabrejko [18].

LEMMA 2.4. Let $F$ be the superposition operator generated by the function $f:[a, b] \times$ $\mathbb{R} \rightarrow \mathbb{R}$. Then $F$ transforms the space $C[a, b]$ into itself and is continuous if and only if the function $f$ is continuous on the set $[a, b] \times \mathbb{R}$.

\section{INTEGRAL OPERATOR OF URYSOHN-STIELTJES TYPE}

Let $I$ be a bounded and closed interval in $\mathbb{R}$. In what follows we will always assume for convenience that $I=[0,1]$.

Denote by $B(I)$ the Banach space consisting of all real bounded functions on the interval $I$ with the supremum norm $\|\cdot\|$. Similarly, $C(I)$ denotes the space of functions being continuous on $I$ with the same norm. Moreover, let $B V(I)$ be the Banach space of real functions of bounded variation on the interval $I$ and normed in the standard way.

Suppose that $g: I \times I \rightarrow \mathbb{R}$ is a given function which is subject to the following conditions: 
(i) the function $t \rightarrow g(t, s)$ is of bounded variation on $I$ for each fixed $s \in I$,

(ii) for every $t \in I$ the function $s \rightarrow g(t, s)$ is of bounded variation on $I$.

Now, let us take a real function $x=x(t)$ being defined and continuous on the interval $I$. Under these assumptions we can consider the function $S x$ defined on $I$ by the formula

$$
(S x)(t)=\int_{0}^{1} x(s) d_{s} g(t, s),
$$

where the symbol $d_{s}$ indicates that the integration is taken with respect to $s$.

Observe that for a fixed $t \in I$ the function $x=x(s)$ is Stieltjes integrable with respect to the function $s \rightarrow g(t, s)$ which is a simple consequence of the criterion of Stieltjes integrability mentioned in the previous section. Thus the function $S x$ is well defined on $I$.

Now we provide a collection of assumptions imposed on the function $g=g(t, s)$ which will be used in the sequel.

(iii) the function $g$ is bounded from above on the set $I \times I$.

Observe that under the assumption (iii) we can consider the function $G: I \rightarrow \mathbb{R}$ defined by

$$
G(s)=\sup _{0 \leq t \leq 1} g(t, s)
$$

We will further assume that

(iv) for each fixed $t \in I$ the function $s \rightarrow G(s)-g(t, s)$ is nondecreasing on the interval $I$,

(v) for all $t_{1}, t_{2} \in I$ such that $t_{1}<t_{2}$ the function $s \rightarrow g\left(t_{2}, s\right)-g\left(t_{1}, s\right)$ is nondecreasing on the interval $I$,

(vi) $g(0, s)=0$ for any $s \in I$,

(vii) the function $t \rightarrow \mathrm{V}_{s=0}^{1} g(t, s)$ is bounded on the interval $I$,

(viii) the functions $t \rightarrow g(t, 1)$ and $t \rightarrow g(t, 0)$ are continuous on $I$

REMARK. Let us pay attention to the fact that from the assumptions (v) and (vi) follows that the function $s \rightarrow g(t, s)$ is nondecreasing on $I$ for each fixed $t \in I$. Obviously in this case the function $G(s)$ is also nondecreasing on $I$.

In order to illustrate the assumptions (iv) and ( $v$ ) and some relationships between these assumptions we provide a few examples (cf. also Remark given above).

EXAMPLE 3.1. Let $g(t, s)=a(t)+b(s)$, where $a$ and $b$ are bounded functions on the interval $I=[0,1]$. Then it is easy to check that $g$ satisfies the assumption (iv).

EXAMPLE 3.2. Take $g(t, s)=a(t) b(s)$, where $a$ is bounded and $b$ is nondecreasing on $I$ Then it is easily seen that $g$ satisfies (iv).

EXAMPLE 3.3. Put $g(t, s)=(t+s)^{2}$ for $t, s \in I$. It may be shown that this function satisfies both assumption (iv) and (v).

EXAMPLE 3.4. Assume that the function $g(t, s)$ is nondecreasing with respect to $t$ and satisfies the assumption (v). Then we can show that $g$ satisfies also the assumption (iv).

EXAMPLE 3.5. Let $g(t, s)=(1-t) s^{2}+t$ for $t, s \in I$. It may be easily shown that $g$ is nondecreasing with respect to both variables $t$ and $s$ but it does not satisfy the assumption (iv).

EXAMPLE 3.6. Let $g(t, s)=a(t) b(s)$ for $t, s \in I$. Suppose that $a$ is decreasing and $b$ is increasing on the interval $I$. Then we can show that $g$ verifies the assumption (iv) but does not verify the assumption (v).

On the other hand if we assume that both $a$ and $b$ are decreasing on $I$ then we may prove that $g$ satisfies the assumption ( $v$ ) but does not satisfy the assumption (iv).

Now we are going to formulate the assumptions describing the properties of the function $u=u(t, s, x)$ which will be considered in our study.

Namely, we assume the following hypotheses. 
(ix) for every $t \in I$ the function $(s, x) \rightarrow u(t, s, x)$ is continuous on the set $I \times \mathbb{R}$,

(x) the function $u$ is continuous on the set $I \times I \times \mathbb{R}$,

(xi) there exists a positive constant $K$ such that

$$
|u(t, s, x)| \leq K(1+|x|)
$$

for all $(t, s, x) \in I \times I \times \mathbb{R}$.

Let us mention that under the assumption (ix) we can consider the function $\omega_{T}(t, \varepsilon)$ which is defined for each $T \geq 0$ and $\varepsilon \geq 0$ in the following way

$$
\begin{gathered}
\omega_{T}(t, \varepsilon)=\sup \{|u(t, p, x)-u(t, q, y)|: p, q \in I,|p-q| \leq \varepsilon, \\
x, y \in \mathbb{R},|x| \leq T,|y| \leq T,|x-y| \leq \varepsilon\} .
\end{gathered}
$$

In what follows we will use the additional assumption concerning the function $u(t, s, x)$ being stronger than the assumption (ix):

(xii) for any $T>0$

$$
\lim _{\varepsilon \rightarrow 0}\left\{\sup _{0 \leq t \leq 1} \omega_{T}(t, \varepsilon)\right\}=0
$$

The last assumption which will be taken into account is the following one.

(xiii) there exists a function $v(t, s)=v: I \times I \rightarrow \mathbb{R}$ which is continuous with respect to $s$ for any $t \in I$ and of bounded variation with respect to $t$ for each $s \in I$ and such that

$$
|u(p, s, x)-u(q, s, x)| \leq|v(p, s)-v(q, s)| \cdot|x|
$$

for all $p, q, s \in I$ and for every $x \in \mathbb{R}$. Moreover, the function $s \rightarrow \underset{t=0}{1} v(t, s)$ is Stieltjes integrable with respect to the function $G(s)$ defined above.

Now we start with the study of the properties of the Urysohn-Stieltjes operator $U$ given by the formula

$$
(U x)(t)=\int_{0}^{1} u(t, s, x(s)) d_{s} g(t, s) \quad(t \in I)
$$

for each real function $x=x(t)$ defined and continuous on the interval $I$.

Throughout this section we will always assume that the functions $g=g(t, s)$ and $u=u(t, s, x)$ satisfy the assumptions (i), (ii), (v), (vi) and (xi).

Other additional assumptions will be quoted in theorems and corollaries which we are going to prove below.

The first property of the operator $U$ defined above is connected with the bounded variation.

THEOREM 3.1. Assume additionally that the hypotheses (iii), (iv), (ix) and (xii) are satisfied. Then the operator $U$ transforms the space $C(I)$ into the space $B V(I)$.

PROOF. Let us fix an arbitrary partition $0=t_{0}<t_{1}<\ldots<t_{n}=1$ of the interval $I$. Then, for any fixed function $x \in C(I)$ we get

$$
\begin{aligned}
& \sum_{i=1}^{n}\left|(U x)\left(t_{\imath}\right)-(U x)\left(t_{i-1}\right)\right| \leq \\
& \leq \sum_{i=1}^{n}\left|\int_{0}^{1} u\left(t_{i}, s, x(s)\right) d_{s} g\left(t_{i}, s\right)-\int_{0}^{1} u\left(t_{\imath-1}, s, x(s)\right) d_{s} g\left(t_{i}, s\right)\right|+ \\
& +\sum_{i=1}^{n}\left|\int_{0}^{1} u\left(t_{i-1}, s, x(s)\right) d_{s} g\left(t_{i}, s\right)-\int_{0}^{1} u\left(t_{i-1}, s, x(s)\right) d_{s} g\left(t_{i-1}, s\right)\right|= \\
& =\sum_{i=1}^{n}\left|\int_{0}^{1}\left[u\left(t_{i}, s, x(s)\right)-u\left(t_{i-1}, s, x(s)\right)\right] d_{s} g\left(t_{i}, s\right)\right|+ \\
& +\sum_{i=1}^{n}\left|\int_{0}^{1} u\left(t_{i-1}, s, x(s)\right) d_{s}\left[g\left(t_{i}, s\right)-g\left(t_{i-1}, s\right)\right]\right| .
\end{aligned}
$$


Hence, in view of Lemmata 2.1 and 2.3 we obtain

$$
\begin{aligned}
& \sum_{i=1}^{n}\left|(U x)\left(t_{2}\right)-(U x)\left(t_{\imath-1}\right)\right| \leq \\
& \leq \sum_{i=1}^{n} \int_{0}^{1} \mid u\left(t_{i}, s, x(s)\right)-u\left(t_{i-1}, s, x(s)\right) \| d_{s}\left(\bigvee_{z=0}^{s} g\left(t_{i}, z\right)\right)+ \\
& +\sum_{i=1}^{n} \int_{0}^{1}\left|u\left(t_{\imath-1}, s, x(s)\right)\right| d_{s}\left(\mathrm{~V}_{z=0}^{s}\left[g\left(t_{\imath}, z\right)-g\left(t_{\imath-1}, z\right)\right]\right)= \\
& =\sum_{i=1}^{n} \int_{0}^{1}\left|u\left(t_{i}, s, x(s)\right)-u\left(t_{2-1}, s, x(s)\right)\right| d_{s} g\left(t_{2}, s\right)+ \\
& +\sum_{i=1}^{n} \int_{0}^{1}\left|u\left(t_{i-1}, s, x(s)\right)\right| d_{s}\left[g\left(t_{i}, s\right)-g\left(t_{i-1}, s\right)\right] \leq \\
& \leq \sum_{i=1}^{n} \int_{0}^{1}\left|v\left(t_{2}, s\right)-v\left(t_{2-1}, s\right)\right| \cdot|x(s)| d_{s} g\left(t_{i}, s\right)+ \\
& +\sum_{i=1}^{n} \int_{0}^{1} K(1+|x(s)|) d_{s}\left[g\left(t_{i}, s\right)-g\left(t_{i-1}, s\right)\right] \leq \\
& \leq\|x\| \sum_{i=1}^{n} \int_{0}^{1}\left|v\left(t_{i}, s\right)-v\left(t_{t-1}, s\right)\right| d G(s)+ \\
& +K(1+\|x\|) \sum_{i=1}^{n} \int_{0}^{1} d_{s}\left[g\left(t_{i}, s\right)-g\left(t_{i-1}, s\right)\right]= \\
& =\|x\| \int_{0}^{1}\left(\sum_{i=1}^{n}\left|v\left(t_{\imath}, s\right)-v\left(t_{\imath-1}, s\right)\right|\right) d G(s)+ \\
& +K(1+\|x\|) \sum_{i=1}^{n} \int_{0}^{1} d_{s}\left[g\left(t_{i}, s\right)-g\left(t_{i-1}, s\right)\right]= \\
& =\|x\| \int_{0}^{1}\left(\mathrm{~V}_{t=0}^{1} v(t, s)\right) d G(s)+K(1+\|x\|)\{[g(1,1)-g(0,1)]-[g(1,0)-g(0,0)]\} .
\end{aligned}
$$

It is not difficult to check that

$$
[g(1,1)-g(0,1)]-[g(1,0)-g(0,0)]>0
$$

which is simple consequence of the assumption (v). This assertion allows us to infer that

$$
\sum_{i=1}^{n}\left|(U x)\left(t_{\imath}\right)-(U x)\left(t_{\imath-1}\right)\right|<\infty .
$$

This completes the proof.

The next object of our study is the continuity of the Urysohn-Stieltjes operator $U$. We have the following result.

THEOREM 3.2. Under the assumptions (vii), (ix) and (xii) the operator $U$ transforms the space $C(I)$ into the space $B(I)$ and is continuous.

PROOF. Let $x \in C(I)$ and let $t \in I$ be fixed arbitrarily. Then, in view of Lemmata 2.1 and 2.2 we get

$$
\begin{aligned}
& |(U x)(t)| \leq \int_{0}^{1}|u(t, s, x(s))| d_{s}\left({\underset{z=0}{\mathrm{~V}}}_{z=0} g(t, z)\right)= \\
& =\int_{0}^{1}|u(t, s, x(s))| d_{s} g(t, s) \leq K \int_{0}^{1}(1+\|x\|) d_{s} g(t, s) \leq \\
& \leq K(1+\|x\|) \sup _{t \in I}[g(t, 1)-g(t, 0)]<\infty .
\end{aligned}
$$


This shows that $U$ acts from the space $C(I)$ into $B(I)$.

Now, fix $\varepsilon>0$ and take an arbitrary function $x \in C(I)$. Denote $T=\|x\|+\varepsilon$.

Further, choose arbitrarily $y \in C(I)$ such that $\|x-y\| \leq \varepsilon$. Then, fixing $t \in I$ and applying Lemmata 2.1 and 2.2 we obtain

$$
\begin{aligned}
& |(U x)(t)-(U y)(t)| \leq \int_{0}^{1}|u(t, s, x(s))-u(t, s, y(s))| d_{s}\left({\underset{z=0}{\mathrm{~V}}}_{z} g(t, z)\right)= \\
& =\int_{0}^{1}|u(t, s, x(s))-u(t, s, y(s))| d_{s} g(t, s) \leq \\
& \leq \int_{0}^{1} \omega_{T}(t, \varepsilon) d_{s} g(t, s) \leq \sup _{t \in I} \omega_{T}(t, \varepsilon) \int_{0}^{1} d_{s} g(t, s) \leq \\
& \leq\left\{\sup _{t \in I} \omega_{T}(t, \varepsilon)\right\} \cdot\left\{\sup _{t \in I}[g(t, 1)-g(t, 0)]\right\} .
\end{aligned}
$$

In view of the assumptions (vii) and (xii) the last estimate implies that the operator $U$ is continuous from $C(I)$ into $B(I)$.

This ends the proof.

Now we are going to formulate a result saying when the Urysohn-Stieltjes operator $U$ is a self-mapping of the space $C(I)$. This result is contained in the following theorem.

THEOREM 3.3. Suppose that the assumptions (viii) and ( $x$ ) are satisfied. Then the operator $U$ transforms the space $C(I)$ into itself.

PROOF. Let us take an arbitrary function $x \in C(I)$. Further, fix arbitrary $t_{1}, t_{2} \in I$. For convenience we will assume that $t_{1}<t_{2}$.

Now, let us denote $T=\|x\|$. Then from the assumption ( $\mathrm{x})$ we infer that the function $u(t, s, x)$ is uniformly continuous on the set $I \times I \times[-T, T]$. This implies that there exists a function $\omega=\omega(\varepsilon)$ such that $\omega(\varepsilon) \rightarrow 0$ as $\varepsilon \rightarrow 0$ and

$$
\left|u\left(t_{2}, s, x(s)\right)-u\left(t_{1}, s, x(s)\right)\right| \leq \omega\left(\left|t_{2}-t_{1}\right|\right)
$$

for each $s \in I$.

Next, using Lemmata 2.1 and 2.2 we deduce the following estimates:

$$
\begin{aligned}
& \left|(U x)\left(t_{2}\right)-(U x)\left(t_{1}\right)\right| \leq \\
& \leq\left|\int_{0}^{1}\left[u\left(t_{2}, s, x(s)\right)-u\left(t_{1}, s, x(s)\right)\right] d_{s} g(t, s)\right|+ \\
& +\left|\int_{0}^{1} u\left(t_{1}, s, x(s)\right) d_{s}\left[g\left(t_{2}, s\right)-g\left(t_{1}, s\right)\right]\right| \leq \\
& \leq \int_{0}^{1}\left|u\left(t_{2}, s, x(s)\right)-u\left(t_{1}, s, x(s)\right)\right| d\left(\mathrm{~V}_{z=0}^{s} g\left(t_{2}, z\right)\right)+ \\
& +\int_{0}^{1}\left|u\left(t_{1}, s, x(s)\right)\right| d\left(\mathrm{v}_{z=0}^{s}\left[g\left(t_{2}, z\right)-g\left(t_{1}, z\right)\right]\right)= \\
& =\int_{0}^{1}\left|u\left(t_{2}, s, x(s)\right)-u\left(t_{1}, s, x(s)\right)\right| d g\left(t_{2}, s\right)+ \\
& +\int_{0}^{1}\left|u\left(t_{1}, s, x(s)\right)\right| d\left(g\left(t_{2}, s\right)-g\left(t_{1}, s\right)\right) .
\end{aligned}
$$


Hence, in view of $(3.1)$ we get

$$
\begin{aligned}
& \left|(U x)\left(t_{2}\right)-(U x)\left(t_{1}\right)\right| \leq \int_{0}^{1} \omega\left(\left|t_{2}-t_{1}\right|\right) d g\left(t_{2}, s\right)+ \\
& +\int_{0}^{1} K(1+|x(s)|) d\left(g\left(t_{2}, s\right)-g\left(t_{1}, s\right)\right) \leq \\
& \leq \omega\left(\left|t_{2}-t_{1}\right|\right) \int_{0}^{1} d g\left(t_{2}, s\right)+K(1+\|x\|) \int_{0}^{1} d\left(g\left(t_{2}, s\right)-g\left(t_{1}, s\right)\right)= \\
& =\omega\left(\left|t_{2}-t_{1}\right|\right)\left\{g\left(t_{2}, 1\right)-g\left(t_{2}, 0\right)\right\}+ \\
& +K(1+\|x\|)\left\{\left[g\left(t_{2}, 1\right)-g\left(t_{1}, 1\right)\right]-\left[g\left(t_{2}, 0\right)-g\left(t_{1}, 0\right)\right]\right\} .
\end{aligned}
$$

The last estimate and the assumptions (viii) and (x) complete the proof.

Observe that as the immediate consequence of the proof of Theorem 3.3 we deduce the following corollary.

COROLLARY 3.1. Under the same assumptions as in Theorem 3.3 the Urysohn-Stieltjes operator $U$ is compact.

Now we formulate a result on the complete continuity of the Urysohn-Stieltjes integral operator $U$. This result can be easily deduced from the conjunction of Theorems 3.2 and 3.3 .

COROLLARY 3.2. Under the assumptions (vii), (viii) and (x) the Urysohn-Stieltjes operator $U$ is a completely continuous mapping of the space $C(I)$ into itself.

REMARK. Let us mention that the assumptions (ix) and (xii) do not appear in Corollary 3.2 since they are covered by the assumption (x). On the other hand the assumptions (ix) and (xii) do not imply the assumption ( $x$ ). Indeed, in order to show this it is enough to take the function $u(t, s, x)=a(t) b(s, x)$, where $b$ is continuous on the set $I \times \mathbb{R}$ while $a$ is bounded but discontinuous on $I$.

\section{REMARKS CONCERNING THE HAMMERSTEIN-STIELTJES INTEGRAL OPERATOR}

In this section we are going to give a few remarks concerning the Hammerstein-Stieltjes integral operator $H$ defined in the following way

$$
(H x)(t)=\int_{0}^{1} k(t, s) f(s, x(s)) d_{s} g(t, s), \quad t \in I .
$$

Obviously the operator $H$ is a particular case of the Urysohn-Stieltjes integral operator $U$ considered in Section 3.

At first let us observe that the collection of the assumptions (i)-(viii) formulated before can be transformed without any change to our situation. On the other hand we have to adopt the assumptions (ix)-(xiii) to the situation of Hammerstein-Stieltjes operator $H$.

Thus, in the sequel of this section we will assume the following hypotheses:

(xiv) the function $f: I \times \mathbb{R} \rightarrow \mathbb{R}$ is continuous and $|f(t, x)| \leq a+b|x|$ for some nonnegative constants $a$ and $b$,

(xv) the function $k(t, s)=k: I \times I \rightarrow \mathbb{R}$ is continuous with respect to $s$ for any $t \in I$. Moreover, $k$ is bounded on the set $I \times I$ i.e. $|k(t, s)| \leq K<\infty$ for all $(t, s) \in I \times I$,

(xvi) the function $k=k(t, s)$ is of bounded variation on the interval $I$ with respect to $t$ for each fixed $s \in I$. Moreover, the function $s \rightarrow \mathrm{V}_{t=0}^{1} k(t, s)$ is Stieltjes integrable on $I$ with respect to the function $G(s)$ defined in Section 3,

$$
(\mathrm{xvii}) \lim _{\varepsilon \rightarrow 0}\left\{\sup _{t \in I}[|k(t, p)-k(t, q)|: p, q \in I,|p-q| \leq \varepsilon]\right\}=0
$$


(xviii) the function $k$ is continuous on the set $I \times I$.

Now, using of some assumptions from the collections (i)-(viii) and (xiv)-(xviii) we can formulate counterparts of the results contained in Theorems 3.1, 3.2, 3.3 or in Corollary 3.2.

For example, in order to formulate counterparts of the results contained in Theorem 3.1 and Corollary 3.2 let us assume that the functions $g(t, s)$ and $f(t, x)$ satisfy the assumptions (i), (ii), (v), (vi) and (xiv). Then we have the following theorems.

THEOREM 4.1. Assume that the functions $g(t, s), f(t, x)$ and $k(t, s)$ satisfy additionally the assumptions (iii), (iv), (xv) and (xvi). Then the Hammerstein-Stieltjes operator $H$ transforms the space $C(I)$ into the space $B V(I)$.

THEOREM 4.2. Under the additional hypotheses (vii), (viii) and (xviii) the HammersteinStieltjes operator $H$ is a completely continuous mapping of the space $C(I)$ into itself.

Obviously in the similar way we can formulate results being analogous to those from Theorems 3.2 and 3.3. We omit easy details.

\section{SOLVABILITY OF INTEGRAL EQUATIONS OF URYSOHN-STIELTJES TYPE}

In this section we will apply the results obtained in the previous sections in the proof of the theorem on the existence of solutions of the Urysohn-Stieltjes integral equation of the form

$$
x(t)=m(t)+\int_{0}^{1} u(t, s, x(s)) d_{s} g(t, s), \quad t \in I .
$$

Let us assume that the functions $u=u(t, s, x)$ and $g=g(t, s)$ involved in the equation (5.1) satisfy the assumptions (i), (ii), (v), (vi), (vii), (viii), (x) and (xi). Moreover, assume that $m . I \rightarrow \mathbb{R}$ is a continuous function.

Then we can formulate the following existence result.

THEOREM 5.1. Suppose that there are satisfied the assumptions listed above. Moreover, assume that $\beta<1$, where $\beta$ is the constant defined by

$$
\beta=K \sup [g(t, 1)-g(t, 0): t \in I] .
$$

Then the equation (5.1) has at least one solution in the space $C(I)$.

PROOF. Observe that in virtue of Theorem 3.3 the operator $F$ generated by the right hand side of the equation (5.1) transforms the space $C(I)$ into itself.

Further, taking into account the assumed hypotheses, for an arbitrary $x \in C(I)$ and for a fixed $t \in I$ we obtain

$$
\begin{aligned}
& |(F x)(t)| \leq|m(t)|+\int_{0}^{1} \mid u\left(t, s, x(s) \mid d_{s} g(t, s) \leq\right. \\
& \leq\|m\|+\int_{0}^{1} K(1+|x(s)|) d_{s} g(t, s) \leq \\
& \leq\|m\|+K(1+\|x\|) \int_{0}^{1} d_{s} g(t, s)=\|m\|+K(1+\|x\|)[g(t, 1)-g(t, 0)] \leq \\
& \leq\|m\|+K(1+\|x\|) \sup [g(t, 1)-g(t, 0): t \in I]=\|m\|+\beta+\beta\|x\| .
\end{aligned}
$$

Hence we get

$$
\|F x\| \leq\|m\|+\beta+\beta\|x\| .
$$

The above estimate implies that the operator $F$ transforms the ball $B(\theta, r)$ into itself provided $r=(\|m\|+\beta)(1-\beta)$. Moreover, in view of Corollary 3.2 the operator $F$ is completely continuous. Thus, by the Schauder fixed point principle we deduce the existence of a solution $x$ of the equation 
(5.1) in the space $C(I)$.

This completes the proof.

Finally let us mention that in the similar way we can formulate an existence theorem for the Hammerstein-Stieltjes integral equation

$$
x(t)=m(t)+\int_{0}^{1} k(t, s) f(s, x(s)) d_{s} g(t, s) .
$$

Namely, assuming the same hypotheses as in Theorem 5.1 with ( $x$ ) and (xi) replaced by (xiv) and (xviii) we have the following theorem.

THEOREM 5.2. Assume that $\beta<1$, where $\beta$ is a constant defined by the formula

$$
\beta=b(\sup [|k(t, s)|: t, s \in I])(\sup [g(t, 1)-g(t, 0): t \in I]) .
$$

Then the equation (5.2) has a solution in the space $C(I)$.

The proof of the above theorem is similar to the proof of Theorem 5.1 and is therefore omitted.

\section{REFERENCES}

[1] BURTON, T.A., "Volterra integral and differential equations," Academic Press, New York 1983.

[2] KRASNOSEL'SKII, M.A., ZABREJKO, P.P., PUSTYL'NIK, J.I. and SOBOLEVSKII, P.J., "Integral operators in spaces of summable functions," Noordhoff, Leyden 1976.

[3] LADDE, G.S., LAKSHMIKANTHAM, V. and ZHANG, B.G., "Oscillation theory of differential equations with deviating argument," Pure and Applied Math., Marcel Dekker 1987.

[4] TRICOMI, F.G., "Integral equations," Int. Publ., New York 1957.

[5] ZABREJKO, P.P., KOSHELEV, A.I., KRASNOSEL'SKII, M.A., MIKHLIN, S.G., RAKOVSCHIK, L.S. and STECSENKO, V.J., "Integral equations," Noordhoff, Leyden 1975.

[6] BITZER, C.W., "Stieltjes-Volterra integral equations," Illinois J. Math. 14 (1970), 434-451.

[7] CHEN, S., HUANG, Q. and ERBE, L.H., "Bounded and zero-convergent solutions of a class of Stieltjes integro-differential equations," Proc. Amer. Math. Soc. 113 (1991), 999-1008.

[8] MACNERNEY, J.S., "Integral equations and semigroups," Illinors J. Math. 7 (1963), 148173.

[9] MINGARELLI, A.B., "Volterra-Stieltjes integral equations and generalized ordinary differential expressions," Lecture Notes in Math., 989, Springer 1983.

[10] BIELECKI, A., "Ordinary differential equations and some of their generalizations," Polzsh Academy of Sciences, Warsaw 1961 (in Polish).

[11] DLOTKO, T., "On some differential equation with retarded argument," Zeszyty Nauk. WSP w Katowicach 4 (1964), 63-72 (in Polish).

[12] DLOTKO, T., "Sur certain équations integro-différentielles du $n$-ieme ordre," Prace Nauk. Univ. Ślask., Katowice 2 (1969), 103-108.

[13] LOVELADY, D.L., "Oscillation and a class of linear delay differential equations," Trans. Amer. Math. Soc. 226 (1977), 345-364.

[14] MYŠKIS, A.D., "Linear differential equations with retarded argument," Nauka, Moscow 1972 (in Russian). (German edition: Deutscher Verlag der Wissenschaften, Berlin 1955).

[15] BANAŚ, J. and DRONKA, J., "Some properties of Fredholm-Stieltjes and HammersteinStieltjes integral operators", Boll. Un. Mat. Ital. (7) 9-B (1995), 203-232.

[16] DUNFORD, N. and SCHWARTZ, J., "Linear operators I," Int. Publ., Leyden 1963.

[17] NATANSON, I.P., "Theory of functions of a real variable," Ungar, New York 1960.

[18] APPELL, J. and ZABREJKO, P.P., "Nonlinear superposition operators," Cambridge Tracts in Math., 95, 1990. 


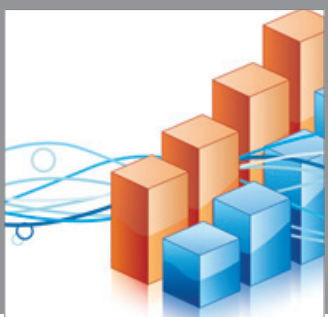

Advances in

Operations Research

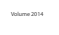

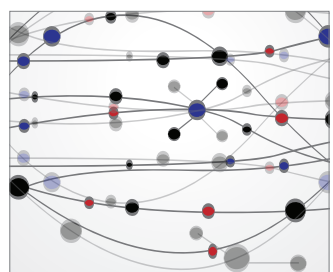

\section{The Scientific} World Journal
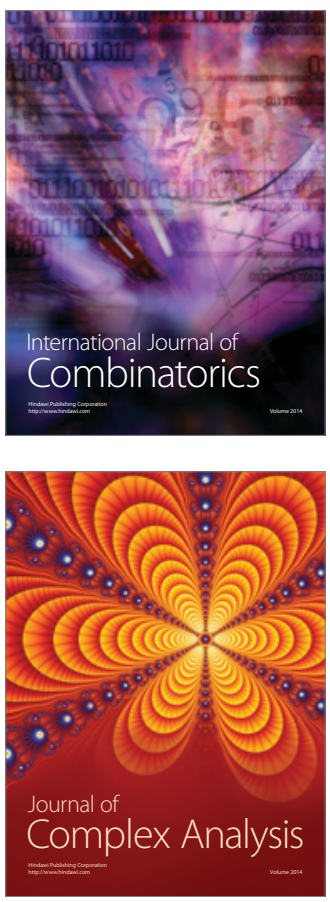

International Journal of

Mathematics and

Mathematical

Sciences
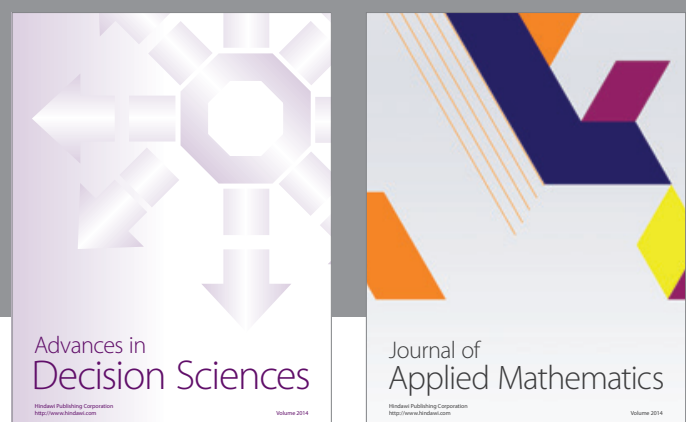

Journal of

Applied Mathematics
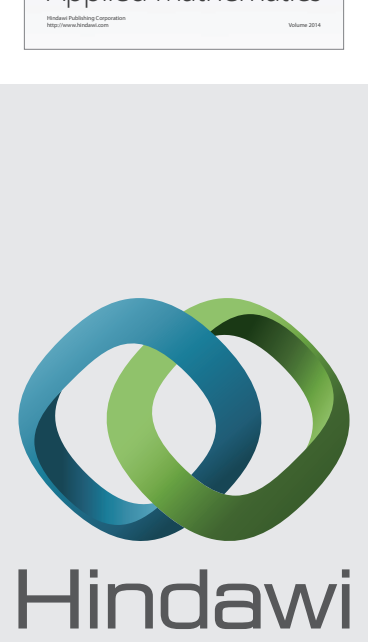

Submit your manuscripts at http://www.hindawi.com
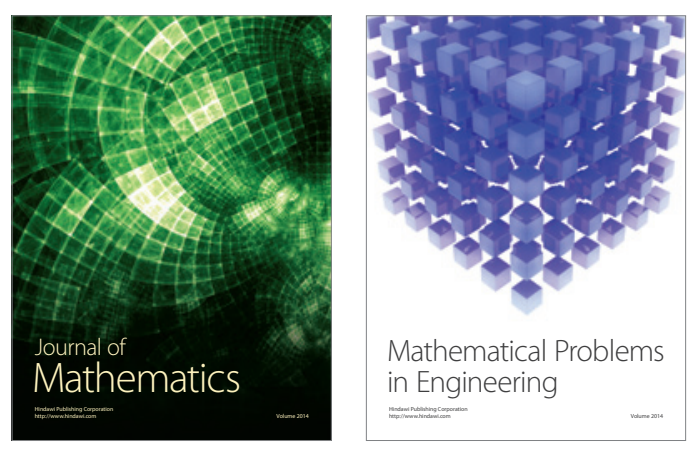

Mathematical Problems in Engineering
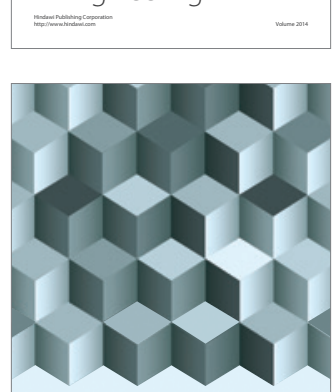

Journal of

Function Spaces
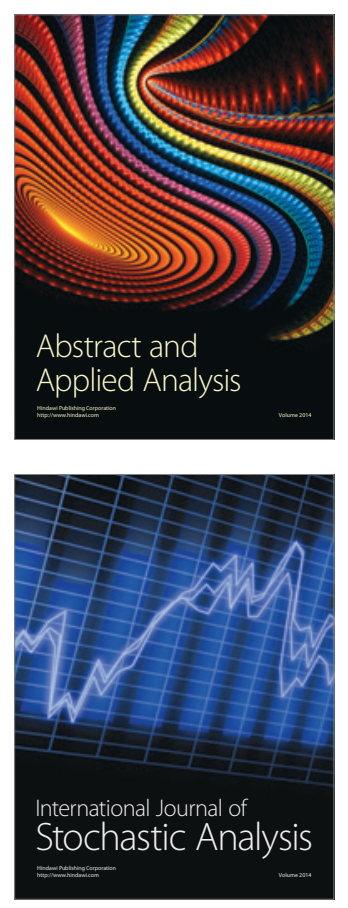

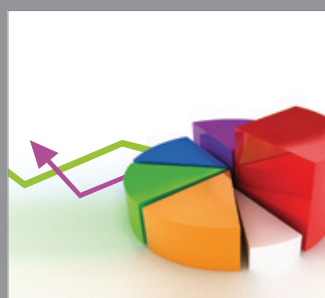

ournal of

Probability and Statistics

Promensencen
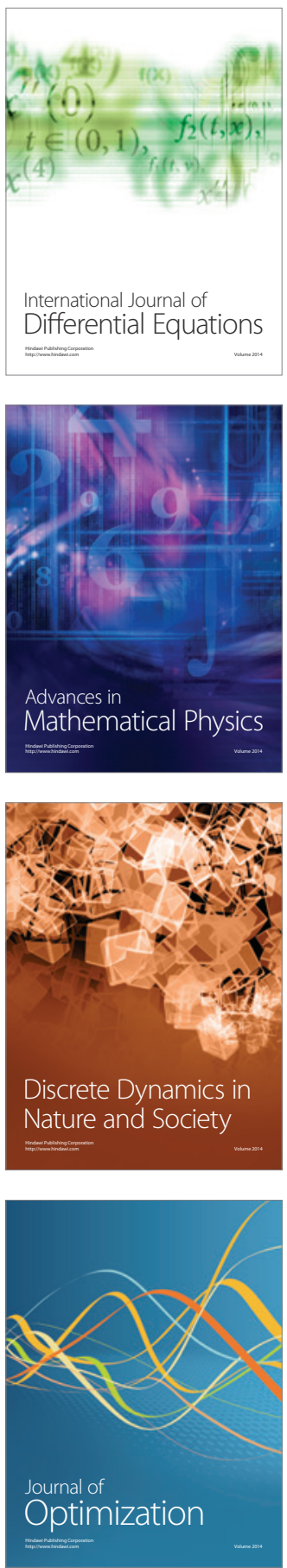